\title{
Inhibitory Effects of Nitrative Stress on the Sulfation of $17 \beta$-Estradiol and 4-Methoxyestradiol by Human MCF 10A Mammary Epithelial Cells
}

\author{
Ying Hui, ${ }^{a, b}$ Tomoko Yasuda, ${ }^{a}$ Shin Yasuda, ${ }^{a}$ Ming-Yih Liu,${ }^{c}$ Yoichi Sakakibara, ${ }^{d}$ Masahito Suiko, ${ }^{d}$ \\ Katherine Ann WALL, ${ }^{a}$ and Ming-Cheh LIU ${ }^{*, a}$ \\ ${ }^{a}$ College of Pharmacy, The University of Toledo; Toledo, OH 43606, U.S.A.: ${ }^{b}$ Department of Obstetric and Gynecology, \\ Beijing Hospital; Beijing, 100730, China: ' National Synchrotron Radiation Research Center; Hsinchu 30076, Taiwan, \\ ROC: and 'Department of Biochemistry and Applied Biosciences, Faculty of Agriculture, University of Miyazaki; \\ Miyazaki 889-2192, Japan. Received December 29, 2009; accepted June 7, 2010
}

\begin{abstract}
Prolonged exposure to high level of estrogen is a known risk factor for breast carcinogenesis. It has been suggested recently that nitrative stress may be an etiologic factor for breast carcinogenesis. Since sulfation plays a major role in the homeostasis of estrogens and their metabolites, we attempted in the present study to find out whether nitrative stress may affect the homeostasis of estrogens through sulfation. Metabolic labeling experiments revealed that the amount of sulfated $17 \beta$-estradiol or 4-methoxyestradiol decreased dramatically in MCF10A mammary epithelial cells incubated in the presence of 3-morpholinosydnonimine (SIN-1) or diethylenetriamine NONOate (DETA NONOate), two nitric oxide donors commonly used to simulate nitrative stress conditions. In searching for the mechanism underlying the decrease of the sulfation of $17 \beta$-estradiol and 4methoxyestradiol, we demonstrated in an in vitro nitration experiment, that the human cytosolic sulfotransferase isoform 1E1 (SULT1E1), a major estrogen-sulfating enzyme, lost its estrogen-sulfating activity proportionately to the degree of nitration on tyrosine residues. Moreover, cell lysates prepared from MCF-10A cells treated with SIN-1 or DETA NONOate also showed much lower 4-methoxyestradiol-sulfating activities, compared with those determined with cell lysate prepared from control MCF-10A cells.
\end{abstract}

Key words $17 \beta$-estradiol; 4-methoxyestradiol; nitrative stress; sulfation; sulfotransferase isoform 1E1

It is widely accepted that elevated level of and prolonged exposure to estrogen plays a central role in breast carcinogenesis. ${ }^{1-3)}$ Estrogen-dependent carcinogenesis may arise from receptor-mediated events that stimulate cellular proliferation and promote tumor progression. ${ }^{4-6)}$ Additionally, estrogens may be hydroxylated to form catecholestrogens (CEs) which then undergo further metabolism to form CEsemiquinones and CE-quinones that are capable of binding to DNA to induce mutations, followed by carcinogenesis. ${ }^{7,8)}$ Whether the human body is equipped with mechanisms for preventing the accumulation of estrogens and CEs, and how such mechanisms may be disrupted under pathophysiological conditions therefore, are important issues for investigation.

Sulfate conjugation is a major pathway in mammals for the biotransformation and excretion of drugs/xenobiotics, as well as the homeostasis of endogenous compounds such as steroid and thyroid hormones, catecholamines, cholesterol, and bile acids. ${ }^{9-11)}$ The responsible enzymes, called the "cytosolic sulfotransferases (SULTs)," catalyze the transfer of a sulfonate group from the active sulfate, $3^{\prime}$-phosphoadenosine 5 '-phosphosulfate (PAPS), to an acceptor substrate compound containing a hydroxyl or an amino group. ${ }^{12)}$ Sulfate conjugation by these enzymes generally result in the inactivation of the substrate compounds and/or increase their water-solubility, thereby facilitating their removal from the body. ${ }^{9-11)}$ In human body, several SULTs, including SULT1A1, SULT1A2, SULT1A3, SULT1C4, SULT1E1, and SULT2A1, have been shown to display sulfating activities toward the two major estrogens, estrone and $17 \beta$-estradiol. ${ }^{13,14)}$ In a recent study, we demonstrated that sulfation of CEs, in addition to estrogens (estrone and $17 \beta$-estradiol), may occur in MCF-7 breast cancer cells and MCF 10A human mammary epithelial cells. ${ }^{15)}$ Enzymatic characterization revealed that, five (SULT1A1, SULT1A2, SULT1A3, SULT1C4, and
SULT1E1) of the eleven known human cytosolic SULTs could use CEs and methoxyestrogens (MEs) as substrates, with SULT1E1 displaying the strongest sulfating activity. It therefore appears that sulfation may serve as a mechanism for the inactivation and/or disposal of CEs and MEs, as well as estrogens.

In recent years, it has been proposed that nitric oxide (NO) and its derivatives produced in inflamed tissues could contribute to the initiation and progression of carcinogenesis. ${ }^{16,17)}$ High concentrations of NO in malignancies are demonstrably mutagenic and several studies have shown that after reaction with oxygen or superoxide, NO forms genotoxic species. ${ }^{18-20)}$ For breast carcinogenesis, a role of oxidative/nitrative stress has also been suggested. ${ }^{21,22)}$ In a study of human primary breast cancers, higher levels of NO synthase (NOS) activity were noted in malignant, compared with benign, tissue and a correlation between tumor grade and NOS levels was observed. ${ }^{23)}$ How elevated NOS activity and the product, NO, may contribute to the etiology of breast carcinogenesis, however, remains unknown. In view of the potential role of sulfation in the inactivation and/or disposal of $\mathrm{CEs} / \mathrm{MEs} / \mathrm{estrogens}$, it is tempting to speculate whether the sulfation of CEs/MEs/estrogens in mammary epithelial cells may be affected under nitrative stress conditions, resulting in the accumulation of CEs/MEs/estrogens which may in turn lead to the initiation and progression of breast carcinogenesis.

We report in this communication findings of a dramatic decrease in the sulfation of $17 \beta$-estradiol and 4-methoxyestradiol in MCF-10A mammary epithelial cells incubated under nitrative stress conditions. Moreover, enzymatic assays showed that purified recombinant SULT1E1, upon treatment with increasing concentrations of peroxynitrite, nitrated SULT1E1 showed proportionately lower levels of $17 \beta$-estra- 
diol-sulfating activity.

\section{MATERIALS AND METHODS}

Materials $17 \beta$-Estradiol $\left(\mathrm{E}_{2}\right)$, adenosine 5 '-triphosphate (ATP), PAPS, Trizma base, 3-( $N$-morpholino)propanesulfonic acid (Mops), inorganic pyrophosphatase, dithiothreitol (DTT), dimethyl sulfoxide (DMSO), tropolone (2-hydroxy2,4,6-cycloheptatrien-1-one), and minimum essential medium (MEM) were obtained from Sigma Chemical Co. (St. Louis, MO, U.S.A.). 3-Morpholinosydnonimine (SIN-1) and diethylenetriamine NONOate (DETA NONOate) were from Cayman Chemical Company (Ann Arbor, MI, U.S.A.). PAP $\left[{ }^{35} \mathrm{~S}\right]$ was synthesized from ATP and carrier-free $\left[{ }^{35} \mathrm{~S}\right]-$ sulfate using the bifunctional human ATP sulfurylase/adenosine $5^{\prime}$-phosphosulfate kinase and its purity determined as previously described. ${ }^{24)}$ Mammary epithelial growth medium (MEGM) was from Lonza Walkersville, Inc. (Walkersville, MD, U.S.A.). HyClone Trypan Blue was from Thermo Scientific (Waltham, MA, U.S.A.). 4-Hydroxyestradiol and 4methoxyestradiol were products of Steraloids, Inc. (Newport, RI, U.S.A.). Carrier-free sodium $\left[{ }^{35} \mathrm{~S}\right]$ sulfate was from ICN Biomedicals Inc. (Irvine, CA, U.S.A.). Cellulose thin-layer chromatography (TLC) plates were from EMD Chem. Inc. (Gibbstown, NJ, U.S.A.). MCF 10A human mammary epithelial cells (ATCC CRL-10317) were from American Type Culture Collection. Non-fat dry milk and Trans-Blot Transfer Medium nitrocellulose membrane $(0.2 \mu \mathrm{m})$ were products of Bio-Rad Laboratories (Hercules, CA, U.S.A.). ECL Advance Western Blotting Kit was purchased from Amersham Biosciences (Piscataway, NJ, U.S.A.). Antibody specific for nitrotyrosine (Clone 1A6; mouse monoclonal immunoglobulin $\left.(\mathrm{Ig}) \mathrm{G}_{2 \mathrm{~b} \kappa}\right)$ and secondary antibody conjugate with horseradish peroxidase (goat anti-mouse immunoglobulin $\mathrm{G}$ (IgG), horseradish peroxidase (HRP)-conjugate, goat polyclonal IgG) were from Upstate (Temecula, CA, U.S.A.). Recombinant human SULT1E1 (estrogen sulfotransferase) was prepared as previously described. ${ }^{25-28)}$ All other chemicals were of the highest grade commercially available.

Treatment of MCF 10A Human Mammary Epithelial Cells with SIN-1 or DETA NONOate MCF 10A cells were routinely maintained, under a $5 \%$ carbon dioxide $\left(\mathrm{CO}_{2}\right)$ atmosphere at $37^{\circ} \mathrm{C}$, in MEGM supplemented with 100 $\mathrm{ng} / \mathrm{ml}$ cholera toxin. For metabolic labeling, confluent $\mathrm{MCF}$ $10 \mathrm{~A}$ cells grown in individual wells of a 24-well culture plate, preincubated in sulfate-free (prepared by omitting streptomycin sulfate and replacing magnesium sulfate with magnesium chloride) MEM for four hours, were labeled with $0.25 \mathrm{ml}$ aliquots of the same medium containing $\left[{ }^{35} \mathrm{~S}\right]$ sulfate $(0.3 \mathrm{mCi} / \mathrm{ml}), 1 \mu \mathrm{M}$ of $17 \beta$-estradiol or 4-hydroxyestradiol, and varying concentrations $(0,0.25,0.5,1,2.5 \mathrm{~mm})$ of SIN-1 or DETA NONOate. At the end of an 18-h labeling, the media were collected and spin-filtered. The filtrates were subjected to the analysis of $\left.{ }^{35} \mathrm{~S}\right]$ sulfated products using the previously established thin-layer electrophoresis (TLE) procedure ${ }^{29)}$ with $5 \%$ acetic acid $/ 0.5 \%$ pyridine $(\mathrm{pH} 3.5)$ as the electrophoresis buffer. After electrophoresis, an autoradiograph was taken from the TLC plate used for the analysis to reveal radioactive spots corresponding to $\left.{ }^{35} \mathrm{~S}\right]$ sulfated metabolites of $17 \beta$-estradiol or 4-methoxyestradiol added to the labeling media. In parallel experiments, MCF-10A cells grown in individual cells were incubated in MEGM and the same concentrations of SIN-1 or DETA NONOate. Upon an 18 -h incubation, the media were removed, and the cells in individual wells were either lysed in $100 \mu \mathrm{l}$ of a lysis buffer (containing $20 \mathrm{M} N$-(2-hydroxyethyl)piperazine- $N$ '-2-ethanesulfonic acid (HEPES)-NaOH, pH 7.0, 1\% Triton X-100, and a protease inhibitor mixture) for examining $17 \beta$-estradiol- or 4-methoxyestradiol-sulfating activity.

Preparation and Western Blot Analysis of Human SULT1E1 Nitrated in Vitro Using Peroxynitrite Purified recombinant human SULT1E1 was treated in vitro with different concentrations (ranging from 0 to $50 \mu \mathrm{M}$ ) of sodium peroxynitrite in $0.15 \mathrm{M}$ sodium bicarbonate solutions. After a 10 -min treatment at $37^{\circ} \mathrm{C}$, the reaction mixtures were immediately chilled on ice, and stored at $-80^{\circ} \mathrm{C}$ until use. To examine the extent of nitration, $10 \mu \mathrm{l}$ aliquots of the treated SULT1E1 solutions were spotted onto a nitrocellulose membrane. The membrane was then blocked using 5\% non-fat milk in phosphate-buffered saline containing $0.1 \%$ Tween 20 (PBST). Afterwards, the membrane was incubated with primary antibody specific for nitrotyrosine at a $1: 1333$ dilution in PBS containing $0.05 \%$ non-fat milk for overnight at $4{ }^{\circ} \mathrm{C}$. Subsequently, secondary antibody conjugated with horseradish peroxidase at a $1: 1333$ dilution in PBST containing $0.5 \%$ non-fat milk for $2 \mathrm{~h}$ at $22^{\circ} \mathrm{C}$. After three washings with PBST, tyrosine-nitrated SULT1E1 protein spot was visualized on an X-ray film using an ECL system.

Sulfotransferase Assay The sulfating activity of untreated or nitrated SULT1E1 or MCF-10A cell lysate was assayed using PAP $\left[{ }^{35} \mathrm{~S}\right]$ as the sulfonate group donor. The standard assay mixture, in a final volume $12.5 \mu 1$, contained $50 \mathrm{~mm}$ of Mops buffer at pH 7.0, $1 \mathrm{~mm}$ DTT, and $14 \mu \mathrm{M}$ PAP $\left[{ }^{35} \mathrm{~S}\right]$. The substrate, $17 \beta$-estradiol $(1 \mu \mathrm{M}$ final concentration) or 4-methoxyestradiol ( $5 \mu \mathrm{M}$ final concentration), dissolved in DMSO at 10 times the final concentration in the assay mixture, was added subsequent to Mops buffer and PAP $\left[{ }^{35} \mathrm{~S}\right]$. Control with DMSO alone was also prepared. The reaction was started by the addition of control or nitrated SULT1E1 $(1.25 \mu \mathrm{g})$ or $2.5 \mu \mathrm{l}$ of MCF-10A cell lysate, allowed to proceed for $10 \mathrm{~min}$ at $37^{\circ} \mathrm{C}$, and stopped by placing the assay mixture-containing thin-wall tube on a heating block, pre-heated to $100{ }^{\circ} \mathrm{C}$, for $2 \mathrm{~min}$. The precipitates were cleared by centrifugation for $1 \mathrm{~min}$, and the supernatant was subjected to the analysis of $\left[{ }^{35} \mathrm{~S}\right]$ sulfated product using TLC with $n$-butanol/isopropanol/88\% formic acid/water ( $3: 1: 1: 1$; by volume) as the solvent system. ${ }^{29)}$ Upon completion of TLC, an autoradiograph was taken from the TLC plate used for the analysis to reveal radioactive spots corresponding to $\left[{ }^{35} \mathrm{~S}\right]$ sulfated $17 \beta$-estradiol or 4 -methoxyestradiol. The radioactive spots were then cut out from the TLC plate, eluted with water, mixed with scintillation fluid, and counted for $\left[{ }^{35} \mathrm{~S}\right]$ radioactivity using a liquid scintillation counter. The counts obtained were used to calculate the specific activities of untreated or nitrated SULT1E1 or MCF$10 \mathrm{~A}$ cell lysate in unit of nmoles sulfated product formed/ $\mathrm{min} / \mathrm{mg}$ purified enzyme. Each assay was performed in triplicate, together with a control without enzyme.

Trypan Blue Exclusion Cell Viability Assay Confluent MCF 10A cells grown in individual wells of a 24-well culture plate were incubated in MEGM containing $0,0.25,0.5$, 1 , and $2.5 \mathrm{~mm}$ of SIN-1 or DETA NONOate. Upon an 18- 
hour incubation, the media were removed and the cells in individual wells were rinsed three times with phosphatebuffered saline (PBS) and incubated in $0.2 \%$ trypan blue in PBS. After a 3-min incubation at room temperature, the cells were rinsed three times with PBS and observed for trypan blue penetration using an inverted microscope.

\section{RESULTS AND DISCUSSION}

The current study was designed to examine the consequence of nitrative stress on the sulfation of $17 \beta$-estradiol and its metabolites in normal and cancerous human mammary epithelial cells and to evaluate the effect of tyrosine nitration, which is known to occur under nitrative stress conditions, on the human SULT1E1, a major enzyme involved in the sulfation of $17 \beta$-estradiol and its metabolites. ${ }^{14,15)}$ As mentioned previously, nitric oxide (NO) and its derivatives produced in inflamed tissues have been proposed to contribute to the initiation and progression of carcinogenesis, ${ }^{16,17)}$ and prolonged exposure to elevated level of estrogens has been cited as a mechanism for breast carcinogenesis. ${ }^{1-6)}$ We were interested in finding out whether under nitrative stress conditions, the sulfation of estrogens and their metabolites in mammary epithelial cells may be affected, thereby leading to the accumulation of these compounds which in turn may trigger the onset and progression of breast carcinogenesis.

Inhibition of the Sulfation of $17 \beta$-Estradiol and 4Methoxyestradiol in MCF-10A Mammary Epithelial Cells by SIN-1 and DETA NONOate In a metabolic labeling experiment, we tested the effects of SIN-1 and DETA NONOate, two NO donors commonly used to simulate nitrative stress conditions, on the sulfation of $17 \beta$-estradiol (a major endogenous estrogen) and 4-hydroxyestradiol (one of the four catecholestrogens) in MCF-10A cells. Confluent cells grown in individual wells of 24 -well plates were labeled with $\left[{ }^{35} \mathrm{~S}\right]$ sulfate in medium containing $5 \mu \mathrm{M} 17 \beta$-estradiol or 4-hydroxyestradiol plus different concentrations (ranging from $0-2.5 \mathrm{~mm}$ ) of SIN-1 or DETA NONOate. As shown in Fig. 1, in the presence of increasing concentrations of SIN-1 or DETA NONOate, there was a dramatic decrease in the generation and release of $\left[{ }^{35} \mathrm{~S}\right]$ sulfated $17 \beta$-estradiol by MCF-10A mammary epithelial cells. In the case of 4-hydroxyestradiol, a similar trend was found for a $\left[{ }^{35} \mathrm{~S}\right]$ sulfated species which, upon thin-layer electrophoresis, migrated at a higher position than $\left[{ }^{35} \mathrm{~S}\right]$ sulfated 4-hydroxyestradiol enzymatically synthesized (figure not shown; ref. 9). The identity of this $\left[{ }^{35} \mathrm{~S}\right]$ sulfated species as $\left[{ }^{35} \mathrm{~S}\right]$ sulfated 4-methoxyestradiol was confirmed in subsequent experiments performed in the presence of tropolone, an inhibitor for catechol- $O$ methyltransferase. ${ }^{9)}$ It therefore appeared that the sulfation of 4-methoxyestradiol derived from the methylation of $17 \mathrm{~b}$ estradiol also decreased dramatically under nitrative stress conditions. Sulfation is generally known to serve as a mechanism for the modulation and homeostasis of estrogens as well as other key endogenous compounds such as thyroid and steroid hormones, as well as catecholamines. ${ }^{10-12)}$ That the sulfation of $17 \beta$-estradiol and 4-methoxyestradiol was suppressed when MCF-10A cells were incubated in the presence of SIN-1 or DETA may imply that under nitrative stress conditions, $17 \beta$-estradiol and 4-methoxyestradiol, as well as other estrogens and their metabolites, may accumulate in the unsulfated form. It is noted in Fig. 1 that both SIN-1 and DETA NONOate inhibited more effectively the sulfation of 4 -methoxyestradiol than that of $17 \beta$-estradiol. The mechanism underlying the differential inhibitory effects of SIN-1 vs. DETA NONOate remains to be clarified. Considering that there are several human SULTs, including SULT1A1, SULT1A2, SULT1A3, SULT1C4, and SULT1E1, that are capable of sulfating $17 \beta$-estradiol and/or 4-methoxyestradiol, ${ }^{15}$ ) it is possible that the differential effects of nitrative stress, as imposed by SIN-1 or DETA NONOate, on the sulfating activity of these SULTs might have resulted in the different extent of inhibition of the sulfation of $17 \beta$-estradiol vs. 4methoxyestradiol. To examine whether, under the experimental conditions adopted, SIN-1 or DETA NONOate may exert cell toxicity thereby leading to the cell death and consequently the decreased level of $17 \beta$-estradiol- or 4methoxyestradiol-sulfating SULT(s), parallel experiments were performed in which SIN-1- or DETA NONOate-treated MCF-10A cells were subjected to trypan blue exclusion test. No significant differences between untreated cells and cells treated with different concentrations of SIN-1 or DETA NONOate were observed. For MCF-10A cells without or with treatment with SIN-1 or DETA NONOate, less than $0.01 \%$ of the cell population showed the penetration of trypan blue. It therefore appears that the inhibition of the sulfation of $17 \beta$-estradiol or 4-methoxyestradiol in SIN-1- or DETA NONOate-treated MCF-10A cells is not due to the cytotoxic effects of SIN-1 or DETA NONOate and, instead,
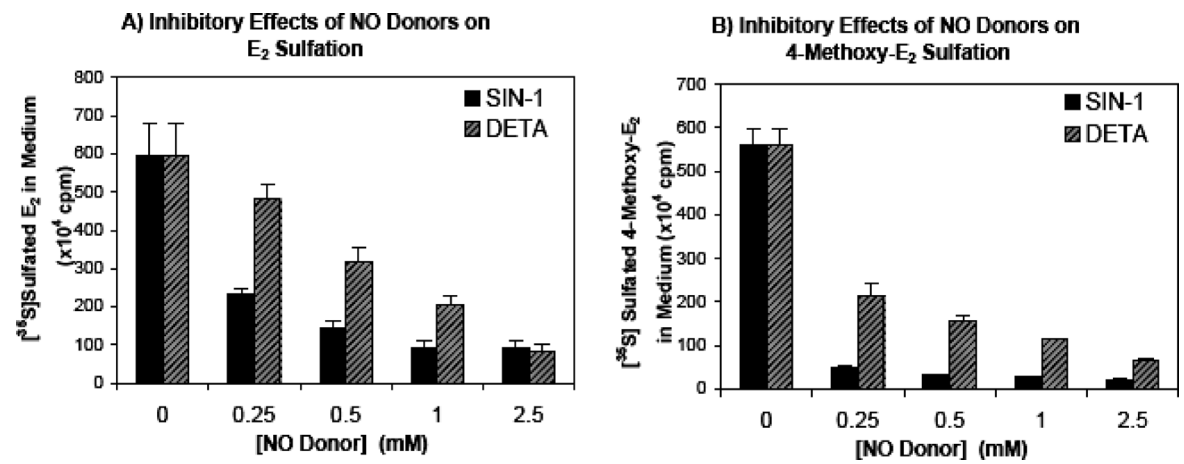

Fig. 1. Generation and Release of $\left[{ }^{35} \mathrm{~S}\right]$ sulfated $17 \beta$-Estradiol $\left(\mathrm{E}_{2}\right)$ and $\left[{ }^{35} \mathrm{~S}\right]$ sulfated 4-Hydroxyestradiol (4-Hydroxy- $\mathrm{E}_{2}$ ) by MCF-10A Human Mammary Epithelial Cells Labeled with ${ }^{35}$ S $]$ sulfate in Medium Containing A) $E_{2}$ or B) 4-Methoxyestradiol (4-Methoxy- $E_{2}$ ) in the Presence of Different Concentrations of SIN-1 or DETA NONOate 
Table 1. Sulfating Activities of Cell Lysates Prepared from MCF-10A Cells Treated with Different Concentrations of SIN-1 or DETA NONOate Using 4-Methoxyestradiol as Substrate ${ }^{a)}$

\begin{tabular}{ccccc}
\hline \hline \multirow{2}{*}{ Control } & \multicolumn{4}{c}{ Specific activity (pmol/min/mg protein) } \\
\cline { 2 - 5 } & $\begin{array}{c}0.25 \mathrm{~mm} \text { SIN-1 } \\
0.5 \mathrm{~mm} \text { SIN-1 }\end{array}$ & $1 \mathrm{~mm} \mathrm{SIN-1}$ & $2.5 \mathrm{~mm}$ SIN-1 \\
\hline $\begin{array}{c}2.56 \pm 0.16 \\
(100 \%)\end{array}$ & $\begin{array}{c}2.18 \pm 1.46 \\
(85.2 \%)\end{array}$ & $\begin{array}{c}1.52 \pm 0.20 \\
(59.4 \%)\end{array}$ & $\begin{array}{c}1.21 \pm 0.06 \\
(47.3 \%)\end{array}$ & $\begin{array}{c}0.65 \pm 0.13 \\
(25.4 \%)\end{array}$ \\
\hline & $0.25 \mathrm{~mm}$ DETA & $0.5 \mathrm{~mm}$ DETA & $1 \mathrm{~mm}$ DETA & $2.5 \mathrm{~mm} \mathrm{DETA}$ \\
\cline { 2 - 5 } & $\begin{array}{c}1.93 \pm 0.12 \\
(75.4 \%)\end{array}$ & $\begin{array}{c}1.87 \pm 0.23 \\
(73.0 \%)\end{array}$ & $\begin{array}{c}1.42 \pm 0.11 \\
(55.5 \%)\end{array}$ & $\begin{array}{c}1.26 \pm 0.13 \\
(49.2 \%)\end{array}$
\end{tabular}

a) The final concentration of 4-methoxyestradiol used in the assay mixture was $5 \mu \mathrm{M} . \quad b)$ Data shown represent means \pm S.D. derived from three experiments.

may be associated with the decrease in the activity of responsible SULT enzyme(s). To obtain supporting evidence for the change in SULT activity, lysates were prepared from untreated MCF-10A cells and cells treated with different concentrations of SIN-1- or DETA NONOate. For reasons yet to be clarified, repeated attempts failed to detect $17 \beta$-estradiolsulfating activity in either untreated cells or cells treated with SIN-1 or DETA NONOate. In contrast, 4-methoxyestradiolsulfating activity was detected in both untreated and SIN-1or DETA NONOate-treated cells. Interestingly, activity data compiled in Table 1 showed that, compared with untreated cells, there was a proportionate decrease in 4-methoxyestradiol-sulfating activity in lysates of MCF-10A cells treated with increasing concentrations of SIN-1 or DETA NONOate. As discussed above, it is possible that elevated levels of estrogenic compounds due to the inhibition of their sulfation by nitrative stress may in turn trigger or contribute to the onset and/or progression of carcinogenesis of these cells. It will be important and interesting to find out whether the suppression of the sulfation of estrogens and their metabolites indeed takes place in pathophysiologcal conditions such as chronic inflammation of local tissues.

Effects of Tyrosine Nitration on the $17 \beta$-Estradiol-Sulfating Activity of SULTE1 The decrease in the capacity of mammary epithelial cells to sulfate $17 \beta$-estradiol and 4methoxyestradiol as described above could have been due to the loss of $17 \beta$-estradiol/methoxyestradiol-sulfating activities of the responsible SULTs under oxidative/nitrative stress conditions simulated in the above-mentioned experiment. It is possible that under oxidative/nitrative stress conditions, estrogen/CE/ME-sulfating SULTs may become nitrated and thereby inactivated. To test this possibility, purified recombinant SULT1E1 was treated in vitro with different concentrations (ranging from 0 to $50 \mu \mathrm{M}$ ) of sodium peroxynitrite. The nitration status of the SULT1E1 thus prepared was examined by Western blot analysis using a monoclonal antibody specific for nitrotyrosine. Figure 2 shows the immunoblot of sodium peroxynitrite-treated SULT1E1 probed using a monoclonal anti-nitrotyrosine antibody. The results revealed an increase in the extent of tyrosine nitration of SULT1E1 treated with increasing concentrations of peroxynitrite. Nitrated SULT1E1 thus prepared was tested for sulfating activity toward $17 \beta$-estradiol. As shown in Table 2, upon treatment with increasing concentrations of peroxynitrite, nitrated SULT1E1 showed proportionately lower levels of $17 \beta$-estra-

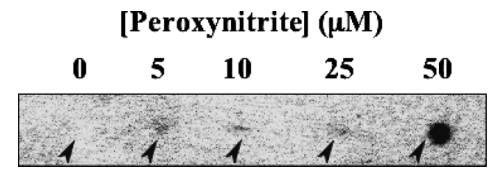

Fig. 2. Immunoblot Analysis of Purified Human SULT1E1 Treated with Different Concentrations of Sodium Peroxynitrite in Vitro

Dot-blotted SULT1E1 preparations were probed using a monoclonal antibody against nitrotyrosine.

Table 2. Specific Activities of Untreated and Peroxynitrite-Treated Human SULT1E1 with $17 \beta$-Estradiol as Substrate ${ }^{a)}$

\begin{tabular}{ccccc}
\hline \hline \multicolumn{5}{c}{$[$ Peroxynitrite] $(\mu \mathrm{M})$} \\
\hline 0 & 5 & 10 & 25 & 50 \\
\hline $1.08 \pm 0.07$ & $0.48 \pm 0.08$ & $0.25 \pm 0.02$ & $0.04 \pm 0.01$ & $0.02 \pm 0.01$ \\
\hline
\end{tabular}

a) Specific activity refers to $\mathrm{nmol}$ substrate sulfated $/ \mathrm{min} / \mathrm{mg}$ purified enzyme. Data represent means \pm S.D. derived from three experiments.

diol-sulfating activity. These results therefore indicated clearly that nitration on tyrosine residues indeed had a dramatic inhibitory effects on the estrogen-sulfating activity of SULT1E1.

In conclusion, we have demonstrated in the present study that nitrative stress, as simulated by the treatment with SIN-1 or DETA, could suppress the sulfation of $17 \beta$-estradiol 4methoxyestradiol, possibly leading to their accumulation in unconjugated, active form which, within the context of cellular physiology, may eventually cause abnormal cell growth and the onset of carcinogenic process. While the underlying mechanism for the suppression of the sulfation of $17 \beta$-estradiol and 4-methoxyestradiol under nitrative stress remains to be fully elucidated, the finding that the loss of estrogensulfating activity of SULT1E1, upon nitration in vitro, may be involved as, at least, part of the overall mechanism.

Acknowledgments This work was supported in part by a startup fund from College of Pharmacy, The University of Toledo.

\section{REFERENCES}

1) Yager J. D., Liehr J. G., Annu. Rev. Pharmacol. Toxicol., 36, 203-232 (1996).

2) Safe S. H., Annu. Rev. Pharmacol. Toxicol., 38, 121-158 (1998).

3) Zhu B. T., Conney A. H., Carcinogenesis, 19, 1-27 (1998).

4) Nandi S., Guzman R. C., Yang J., Proc. Natl. Acad. Sci. U.S.A., 92, 3650-3657 (1995)

5) Castles C. G., Fuqua S. A. W., "Hormone-Dependent Cancer," ed. by Pasqualini J. R., Katzenellebogen B. S., Marcel Dekker, New York, 1996, pp. $81-105$.

6) Preston-Martin S., Pike M. C., Ross R. K., Jones P. A., Henderson B. E., "Cell Proliferation and Chemical Carcinogenesis, International Symposium,” Research Triangle Park, NC, 1992.

7) Liehr J. G., Polycyclic Aromatic Compounds, 6, 229-239 (1994).

8) Cavalieri E. L., Stack D. E., Devanesan P. D., Todorovic R., Dwivedy I., Higginbothan S., Johannson S. L., Patil K. D., Gross M. L., Gooden J. K., Ramanathan R., Cerny R. L., Rogan E. G., Proc. Natl. Acad. Sci. U.S.A., 94, 10937-10942 (1997).

9) Mulder G. J., Jakoby W. B., "Drug Metabolism," ed. by Mulder G. J., Jakoby W. B., Taylor \& Francis, London, 1990, pp. 107-161.

10) Falany C., Roth J. A., "Human Drug Metabolism; from Molecular Biology to Man," ed. by Jeffery E. H., CRC Press, Boca Raton, 1993, pp. $101-115$. 
11) Weinshilboum R., Otterness D., "Conjugation-Deconjugation Reactions in Drug Metabolism and Toxicity," ed. by Kaufmann F. C., Springer-Verlag, Berlin, 1994, pp. 45-78.

12) Lipmann F., Science, 128, 575-580 (1958).

13) Raftogianis R., Creveling C., Weinshilboum R., Weisz J., J. Natl. Cancer Inst. Monogr., 27, 113-124 (2000).

14) Yasuda S., Suiko M., Liu M.-C., J. Biochem. (Tokyo), 137, 401-406 (2005).

15) Hui Y., Yasuda S., Liu M.-Y., Wu Y.-Y., Liu M.-C., Biol. Pharm. Bull., 31, 769-773 (2008)

16) Lithgow D., Covington C., Biol. Res. Nurs., 7, 118-129 (2005).

17) Bartsch H., Nair J., Langenbecks, Arch. Surg., 391, 499-510 (2006).

18) Felley-Bosco E., Cancer Metastasis Rev., 17, 25-37 (1998).

19) Bing R. J., Miyataka M., Rich K. A., Hanson N., Wang X., Slosser H. D., Shi S. R., Clin. Cancer Res., 7, 3385-3392 (2001).

20) Hofseth L. J., Saito S., Hussain S. P., Espey M. G., Miranda K. M., Araki Y., Jhappan C., Higashimoto Y., He P., Linke S. P., Quezado M. M., Zurer I., Rotter V., Wink D. A., Appella E., Harris C. C., Proc. Natl. Acad. Sci. U.S.A., 100, 143-148 (2003).
21) Kang D. H., AACN Clin. Issues, 13, 540-549 (2002).

22) Gago-Dominguez M., Jiang X., Castelao J. E., Breast Cancer Res., 9, 201 (2007).

23) Thomsen L. L., Miles D. W., Happerfield L., Bobrow L. G., Knowles R. G. Moncada S., Br. J. Cancer, 72, 41-44 (1995).

24) Yanagisawa K., Sakakibara Y., Suiko M., Takami Y., Nakayama T., Nakajima H., Takayanagi K., Natori Y., Liu M.-C., Biosci. Biotechnol. Biochem., 62, 1037-1040 (1998).

25) Pai T. G., Sugahara T., Suiko M., Sakakibara Y., Xu F., Liu M.-C., Biochim. Biophys. Acta, 1573, 165-170 (2002).

26) Sakakibara Y., Takami Y., Nakayama T., Suiko M., Liu M.-C., J. Biol. Chem., 273, 6242-6247 (1998).

27) Sakakibara Y., Yanagisawa K., Katafuchi J., Ringer D. P., Takami Y., Nakayama T., Suiko M., Liu M.-C., J. Biol. Chem., 273, 3392933935 (1998).

28) Sakakibara Y., Suiko M., Pai T. G., Nakayama T., Takami Y., Katafuchi J., Liu M.-C., Gene, 285, 39—47 (2002).

29) Liu M.-C., Lipmann F., Proc. Natl. Acad. Sci. U.S.A., 81, 3695-3698 (1984). 